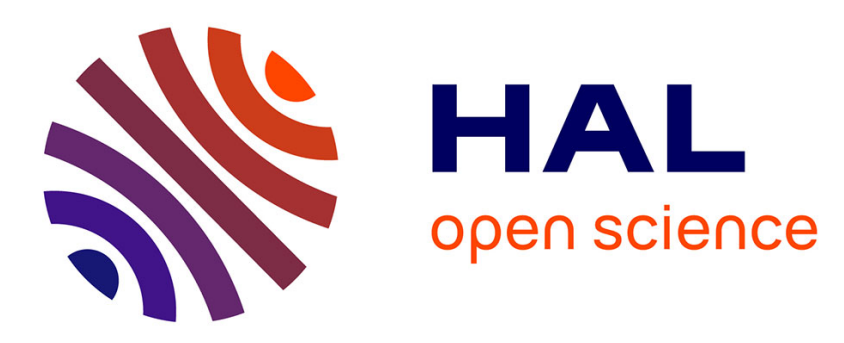

\title{
A single-molecule approach to explore binding, uptake and transport of cancer cell targeting nanotubes
}

Constanze Lamprecht, Birgit Plochberger, Verena Ruprecht, Stefan Wieser, Christian Rankl, Elena Heister, Barbara Unterauer, Mario Brameshuber, Jürgen Danzberger, Petar Lukanov, et al.

\section{To cite this version:}

Constanze Lamprecht, Birgit Plochberger, Verena Ruprecht, Stefan Wieser, Christian Rankl, et al.. A single-molecule approach to explore binding, uptake and transport of cancer cell targeting nanotubes. Nanotechnology, 2014, vol. 25 ( $\mathrm{n}^{\circ} 12$ ), pp.125704. 10.1088/0957-4484/25/12/125704 . hal-01132418

\author{
HAL Id: hal-01132418 \\ https://hal.science/hal-01132418
}

Submitted on 17 Mar 2015

HAL is a multi-disciplinary open access archive for the deposit and dissemination of scientific research documents, whether they are published or not. The documents may come from teaching and research institutions in France or abroad, or from public or private research centers.
L'archive ouverte pluridisciplinaire HAL, est destinée au dépôt et à la diffusion de documents scientifiques de niveau recherche, publiés ou non, émanant des établissements d'enseignement et de recherche français ou étrangers, des laboratoires publics ou privés. 


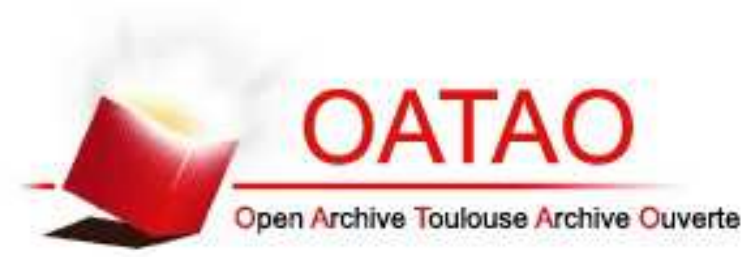

\section{Open Archive TOULOUSE Archive Ouverte (OATAO)}

OATAO is an open access repository that collects the work of Toulouse researchers and makes it freely available over the web where possible.

This is an author-deposited version published in : http://oatao.univ-toulouse.fr/ Eprints ID : 13633

To link to this article : DOI:10.1088/0957-4484/25/12/125704 URL : http://dx.doi.org/10.1088/0957-4484/25/12/125704

\section{To cite this version :}

Lamprecht, Constanze and Plochberger, Birgit and Ruprecht, Verena and Wieser, Stefan and Rankl, Christian and Heister, Elena and Unterauer, Barbara and Brameshuber, Mario and Danzberger, Jürgen and Lukanov, Petar and Flahaut, Emmanuel and Schütz, Gerhard and Hinterdorfer, Peter and Ebner, Andreas A singlemolecule approach to explore binding, uptake and transport of cancer cell targeting nanotubes. (2014) Nanotechnology, vol. $25\left(\mathrm{n}^{\circ}\right.$ 12). p. 125704. ISSN 0957-4484

Any correspondance concerning this service should be sent to the repository administrator: staff-oatao@ listes-diff.inp-toulouse.fr 


\title{
A single-molecule approach to explore binding, uptake and transport of cancer cell targeting nanotubes
}

\author{
C Lamprecht ${ }^{1,2,10}$, B Plochberger ${ }^{3}$, V Ruprecht $^{4}$, S Wieser $^{4}$, C Rankl $^{5}$, \\ E Heister $^{6}$, B Unterauer ${ }^{1}$, M Brameshuber ${ }^{3}$, J Danzberger ${ }^{1}$, P Lukanov ${ }^{7}$, \\ E Flahaut $^{7,8}$, G Schütz $^{3}$, P Hinterdorfer ${ }^{1,9}$ and A Ebner ${ }^{1,10}$ \\ ${ }^{1}$ Institute of Biophysics, Johannes Kepler University, A-4020 Linz, Austria \\ ${ }^{2}$ Institute of Materials Science, Christian-Albrechts-University of Kiel, D-24114 Kiel, Germany \\ ${ }^{3}$ Institute of Applied Physics, University of Technology Vienna, A-1040 Vienna, Austria \\ ${ }^{4}$ Institute of Science and Technology Austria, A-3400 Klosterneuburg, Austria \\ ${ }^{5}$ Agilent Technologies GmbH, A-4020 Linz, Austria \\ ${ }^{6}$ Faculty of Health and Medical Sciences, University of Surrey, Guildford GU2 7XH, UK \\ ${ }^{7}$ University of Toulouse; UPS, INP; Institut Carnot Cirimat, F-31062 Toulouse cedex 9, France \\ ${ }^{8}$ CNRS; Institut Carnot Cirimat, F-31062 Toulouse, France \\ ${ }^{9}$ Center for Advanced Bioanalysis, A-4020 Linz, Austria
}

E-mail: cla@tf.uni-kiel.de and andreas.ebner@jku.at

\begin{abstract}
In the past decade carbon nanotubes (CNTs) have been widely studied as a potential drug-delivery system, especially with functionality for cellular targeting. Yet, little is known about the actual process of docking to cell receptors and transport dynamics after internalization. Here we performed single-particle studies of folic acid (FA) mediated CNT binding to human carcinoma cells and their transport inside the cytosol. In particular, we employed molecular recognition force spectroscopy, an atomic force microscopy based method, to visualize and quantify docking of FA functionalized CNTs to FA binding receptors in terms of binding probability and binding force. We then traced individual fluorescently labeled, FA functionalized CNTs after specific uptake, and created a dynamic 'roadmap' that clearly showed trajectories of directed diffusion and areas of nanotube confinement in the cytosol. Our results demonstrate the potential of a single-molecule approach for investigation of drug-delivery vehicles and their targeting capacity.
\end{abstract}

Keywords: AFM, molecular recognition, force spectroscopy, carbon nanotubes, particle tracking

\section{Introduction}

Carbon nanotubes (CNTs) have attracted much attention as potential multipurpose drug-delivery systems, owing to

10 The authors declare no conflict of interest. their ability to cross cell membranes [1,2], and their exceptionally high surface area that allows attachment of multiple copies of cell targeting molecules [3-5]. A number of groups previously demonstrated successful targeting of cancer cells with folic acid (vitamin $\mathrm{B}_{9}$ ) functionalized CNTs 
[6-8], the delivery of active chemotherapeutic drugs [9, 10], and concentration dependent clearance of CNTs by healthy cells [11]. Selective targeting of certain cancer types using folic acid (FA) has proven to be an effective strategy [12-14]. The uptake is mediated by folic acid binding receptors (FR), which are significantly upregulated in a broad spectrum of cancers [15]. Based on this previous work, our aim is to explore the mechanism of FA mediated CNT targeting on a single nanotube level. Applying single-molecule techniques we want to investigate the first step of CNT binding to the cell surface, past the transport route through the cytosol and final intracellular localization.

In order to directly visualize and quantify the binding of FA functionalized CNTs to cell surface receptors, we employed molecular recognition force spectroscopy (MRFS), an atomic force microscopy (AFM) based method that enables characterization of binding events and binding forces on a single ligand-receptor bond level with Piconewton resolution [16-19]. In MRFS targeting molecules are attached to the apex of the AFM-tip, which is repeatedly brought into contact with receptors on a substrate. When the tip is pulled away from the surface an increasing force is exerted on previously formed bonds and rupture events can be detected. We have bound CNTs covalently to AFM-tips and subsequently functionalized them with FA. The tips where then probed against life carcinoma cells under physiological conditions.

Using single-particle tracking (SPT) [20, 21], we analyzed the paths of CNTs inside the cell after FA mediated uptake. SPT provides a wealth of information about diffusional properties by looking at the mean square displacement (MSD) over time [22, 23] from trajectories of individual particles. To track CNTs inside the cell we employed a fluorescence microscopy technique termed highly inclined laminated optical sheet (HILO) [24]. HILO works at a sub-critical incident angle compared to the well known total internal reflection fluorescence (TIRF) technique, thus enabling 3D fluorescence imaging. For CNT labeling we adapted an uncomplicated but efficient non-covalent functionalization procedure using amine terminated poly(ethylene glycol) lipids that prevents non-specific uptake of CNTs, and allows for stable attachment of the targeting ligand FA and a fluorescent label via the amino group at the same time $[6,8]$.

\section{Methods}

\subsection{Cell culture}

T24 cells (epithelial, urinary bladder carcinoma) were cultured in RPMI 1640 medium (E15-848), containing 10\% fetal calf serum, supplemented with $1 \%$ penicillin/streptomycin, and $1 \%$ 1 M HEPES (all: PAA Laboratories, Vienna, Austria) in an incubator at $37^{\circ} \mathrm{C}$ and $5 \% \mathrm{CO}_{2}$ level. After two passages the medium was changed to RPMI 1640 FA-free (Invitrogen). Cells were passaged at least four rounds in FA-free medium to prevent saturation of FR on the surface of the cells [25]. As control, T24 were cultured in normal RPMI 1640 (10\% fetal calf serum, $1 \%$ penicillin/streptomycin, and 1\% $1 \mathrm{M}$ HEPES) with further supplement of $2 \mu \mathrm{M}$ FA $6 \mathrm{~h}$ prior to AFM MRFS or SPT, respectively. For measurements cells were seeded on glass slides in petri dishes and used at $40-60 \%$ confluence.

\subsection{AFM-tip chemistry}

$\mathrm{Si}_{3} \mathrm{~N}_{4}$ AFM cantilevers with $0.01-0.03 \mathrm{~N} \mathrm{~m}^{-1}$ nominal spring constant (Bruker, MSCT) were functionalized with 3-glycidoxypropyltrimethoxysilane (GPS). A mixture of ethanol/ $\mathrm{H}_{2} \mathrm{O}(\mathrm{v} / \mathrm{v}=95 / 5)$, adjusted to $\mathrm{pH} 4.5$ adding acetic acid, and 2 vol\% GPS, was stirred for ten minutes for silanol formation and added to chloroform cleaned cantilevers. After $8 \mathrm{~h}$ at room temperature (RT), cantilevers were rinsed with n-hexane, dried and placed in a vacuum-desiccator for $24 \mathrm{~h}$ on blue gel for curing. Then, cantilevers were tempered to $50^{\circ} \mathrm{C}$ and incubated with a small droplet of melted $\mathrm{PEG}_{20}$-diamine for $4 \mathrm{~h}$. Afterwards, they were rinsed with a mixture of chloroform/methanol/acetic acid ( $/ \mathrm{v} / \mathrm{v}=$ $70 / 30 / 4)$ and dried before immersion in a solution of EDC 1-ethyl-3-(3-dimethylamino-propyl carbodiimide) and NHS ( $N$-hydroxysuccinimide) (12 $\mu \mathrm{M}$ each) in water-free DMF (dimethylformamide), to which $150 \mu \mathrm{l}$ (300 $\mu \mathrm{l}$ for high number on AFM-tip) of oxidized double walled carbon nanotubes (oxDWNTs, see supporting information stacks.iop. org/Nano/25/125704/mmedia) in MQ water at a concentration of $\sim 0.1 \mathrm{mg} \mathrm{ml}^{-1}$, and $5 \mathrm{vol} \%$ Huenig-base were added. After two hours the tips were transferred to a fresh solution of EDC/NHS (12 $\mu \mathrm{M}$ each) in water-free DMF with the addition of $\mathrm{EG}_{2}$-diamine (diethyleneglycol-diamine) and $5 \mathrm{vol} \%$ Huenig-base for another two hours. Then, tips were immersed in a solution of EDC/NHS (12 $\mu \mathrm{M}$ each) in chloroform with addition of FA $\left(1 \mathrm{mg} \mathrm{ml}^{-1}\right)$ and 5 vol\% Huenig-base again for $2 \mathrm{~h}$. Finally, they were cleaned in chloroform, dried, and stored under argon at $4{ }^{\circ} \mathrm{C}$ for 2-3 days before use.

\subsection{AFM force measurements}

Experiments were performed on a PicoPlus AFM (Agilent Technologies, Chandler, AZ) at RT. Cells were maintained in Hank's buffered saline solution (HBSS). Up to 800 forcedistance curves (approach-retraction cycles) were recorded on the surface of each cell. The pulling rate was set to $4000 \mathrm{~nm} \mathrm{~s}^{-1}$. Between 4 and 6 cells were sampled per CNT-FA functionalized cantilever. Data analysis (cantilever spring constants and FDC analysis) was done using in-house algorithms implemented in MATLAB (MathWorks). For spring constant determination the 'thermal noise' method was used [26].

\subsection{CNT functionalization for cell uptake}

For cell uptake oxDWNTs were functionalized with FA and a fluorescent dye adapting a non-covalent scheme [27] based on DSPE-PEG $2000-\mathrm{NH}_{2}$ (2-distearoyl-sn-glycero-3-phospho ethanolamine- $N$-[amine(PEG2000)]) [6, 8]. DSPE-PEG $2000^{-}$ $\mathrm{NH}_{2}$ was conjugated to FA according to [6]. FA (Sigma Aldrich) (3.5 mM) and $5 \mathrm{mM}$ EDC were added to a solution of $0.35 \mathrm{mM}$ DSPE-PEG-NH $\mathrm{N}_{2}$ in $10 \mathrm{mM}$ phosphate buffer at $\mathrm{pH}$ 7.5. After reaction, the solution was dialyzed against PBS (MW cutoff $14000 \mathrm{Da}$ ) for 2 days with frequent buffer exchange to remove unreacted FA and EDC. Alexa647 dye was coupled to DSPE-PEG $2000-\mathrm{NH}_{2}$ as follows: $10 \mathrm{mg}$ of DSPE-PEG $_{2000}-\mathrm{NH}_{2}$ were dissolved in $1.5 \mathrm{ml}$ of $0.1 \mathrm{M}$ carbonate buffer solution ( $\mathrm{pH} 8.0$ ), and $1 \mathrm{mg}$ of Alexa 
Fluor $^{\mathrm{R}}$ 647-succinimidyl ester (dissolved in DMSO (dimethyl sulfoxide)) was added. The mixture was allowed to react overnight at RT under protection from light. Purification was done by gel chromatography. Labeling was confirmed by NMR yielding a ratio of 1:20 for DSPE-PEG 2000 -Alexa647 to unlabeled DSPE-PEG $2000-\mathrm{NH}_{2}$. A mixture of $1.74 \mathrm{mg}$ DSPE-PEG $2000-\mathrm{NH}_{2} /$ Alexa647 and $0.26 \mathrm{mg} \mathrm{DSPE}_{2} \mathrm{PEG}_{2000^{-}}$ FA in $2 \mathrm{ml}$ MQ water was made with a final ratio of 20:1:3 for DSPE-PEG $2000-\mathrm{NH}_{2} /$ Alexa647/FA.

For non-covalent functionalization $1 \mathrm{mg}$ oxDWNT powder was added to $2 \mathrm{ml}$ of Millipore water and sonicated (Bandelin Sonoplus GM70 tip sonicator, MS 73 microtip, at $40 \%$ power) for 30 min under cooling. Right after sonication the DSPE-PEG $2000-\mathrm{NH}_{2} /$ Alexa647/FA mixture was added, and the solution briefly sonicated for $2 \mathrm{~min}$ in a mild bath sonicator. The suspension was kept shaking at $4{ }^{\circ} \mathrm{C}$ overnight with protection from light, and then diluted to a volume of $10 \mathrm{ml}$. After centrifugation at $14000 \mathrm{~g}$ for $15 \mathrm{~min}$ the supernatant was carefully extracted and filtered several times through centrifugal filters (microsep, $1000 \mathrm{kD}$ cutoff, Pall) to remove excess DSPE-PEG $2000-\mathrm{NH}_{2} /$ Alexa647/FA. The remaining oxDWNT-DSPE- $\mathrm{PEG}_{2000}-\mathrm{NH}_{2} / \mathrm{Alexa647/FA}$ were resuspended in $2 \mathrm{ml} \mathrm{MQ}$ water and kept at $4{ }^{\circ} \mathrm{C}$ under protection from light until further use.

\subsection{CNT delivery to cells}

FA deprived and FA saturated T24 cells (see above) were incubated with oxDWNT-DSPE-PEG $2000-\mathrm{NH}_{2} /$ Alexa647/FA for $8-10 \mathrm{~h}$ adding $80 \mu \mathrm{l}$ of the CNT suspension into the petri-dish containing $2 \mathrm{ml}$ of FA-free or FA-enriched medium. Cells were kept in the incubator during incubation. Before mounting samples on the microscope the cells were washed carefully to remove free CNTs. Fluorescence imaging was done in HBSS buffer at room temperature. Treatment of oxDWNT-DSPE-PEG $2000-\mathrm{NH}_{2}$ /Alexa647/FA incubating (incubation time $9 \mathrm{~h}$ ) cells with nocodazole (Sigma) was done adding the microtubule depolymerizing agent at a concentration of $10 \mu \mathrm{g} \mathrm{ml}^{-1}$ to the cells $1 \mathrm{~h}$ prior to fluorescence experiments. Several concentrations within the range of $0.04-10 \mu \mathrm{g} \mathrm{ml}^{-1}$, as given by the manufacturer, were tested. Only the highest concentration of $10 \mu \mathrm{g} \mathrm{ml}^{-1}$ yielded visible results in destabilizing the microtubule network in T24 cells.

\subsection{Fluorescence microscope}

Experiments were carried out on an inverted light microscope (Zeiss Axiovert 200) with a $100 \times \mathrm{NA}=1.46$ oil TIRFM planAPOCHROMAT objective (Olympus, Hamburg Germany). Samples were illuminated in objective-type total internal reflection configuration (TIRF) or highly inclined and laminated optical sheet (HILO) configuration via the epiport using $647 \mathrm{~nm}$ light from a $\mathrm{Kr}^{+}$-Laser (Stabilite 2017-KR, Spectra

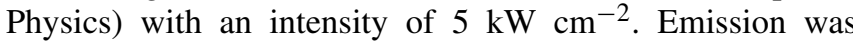
recorded on a thermo electrically cooled CCD-camera (Andor iXon Du-897 BV, South Windsor USA) after passing a filter (FITC/Cy5, Semrock). An acoustic-optical modulator (1205C, Isomet, Springfield, VA, USA) was used for precise control of illumination protocols. Sequences of 100-200 frames were recorded at $5 \mathrm{~ms}$ illumination $\left(t_{\mathrm{ill}}\right)$ and typically $100-500 \mathrm{~ms}$ delay $\left(t_{\text {delay }}\right)$ between consecutive frames.

\subsection{CNT tracking data analysis}

Images were analyzed using in-house algorithms implemented in MATLAB (MathWorks). Individual diffraction limited signals were selected and fitted with a Gaussian intensity profile, yielding the single-molecule position $\vec{r}(t)$ with an accuracy $\sigma_{x y}$; we determined an accuracy of $\sim 50 \mathrm{~nm}$. Single-molecule trajectories were reconstructed [23], and the mean square displacement $\operatorname{MSD}\left(t_{\mathrm{lag}}\right)=\left\langle\left(\vec{r}\left(t+t_{\mathrm{lag}}\right)-\vec{r}(t)\right)^{2}\right\rangle$ was calculated as function of the time-lag $t_{\text {lag }}=t_{\text {ill }}+t_{\text {delay }}$.

The resulting trajectories were plotted as mean square displacement versus time (MSD versus $t_{\mathrm{lag}}$ ) and fitted using the following fit functions:

(a) confined diffusion within a domain of radius $R$ (diffusion constant $D_{\text {micro }}$ ) [23]:

$$
\operatorname{MSD}_{\text {circle }}=R^{2}\left(1-\exp \left(-\frac{4 D_{\text {micro }} t_{\text {lag }}}{R^{2}}\right)\right)+4 \sigma_{x y}^{2}
$$

(b) directed motion [28]:

$$
\mathrm{MSD}=v^{2} t^{2}+4 \sigma_{x y}^{2}
$$

\section{Results}

\subsection{AFM-tip functionalization and molecular recognition force spectroscopy on T24 cells}

Though functionalization of AFM cantilevers for MRFS is a well established procedure [29, 30] attachment of CNTs is a novel approach and a new protocol had to be developed (figure 1(C)). First, commercial silicon nitride cantilevers were coated with GPS (3-glycidoxypropyltrimethoxy silane) to provide high numbers of functional groups on the surface, to which $\mathrm{PEG}_{20}$-diamine linkers (stretched linker length $\sim 6 \mathrm{~nm}$ ) were bound in a second step. To the extending amino groups of the PEG-chains oxidized double walled carbon nanotubes (oxDWNTs) [31] were bound via activated carboxyl groups present on the nanotube surface. In a fourth step a short PEG-linker ( $\mathrm{EG}_{2}$ diamine) was coupled to the remaining accessible activated $\mathrm{COOH}$ groups on the oxDWNTs allowing the final coupling of the targeting molecule FA. When using high amounts of oxDWNTs a dense packing of nanotubes was obtained as shown in figure 1(A). As for MRFS experiments ideally only a single tube should be attached to the tip apex, we reduced the amount of oxDWNT solution in the coupling procedure adequately (figure 1(B)). Nevertheless, at present we lack the possibility to accurately control the exact number of nanotubes at the very end of the tip and we expect to find rather a small intertwined bundle instead of an individual tube (figure 1(B), inset).

MRFS experiments were done on live T24 (urinary bladder carcinoma) cells. The cells were deprived of FA for an extended period to prevent saturation of FA binding receptors (FR) by free agonist [32, 33]. Figure 2 shows so-called force-distance curves (FDCs) in the representation of acting force versus tip-surface separation (distance). In more detail: the CNT-FA functionalized AFM-tip vertically approaches the sample surface (black curve, starting from the right), the force 

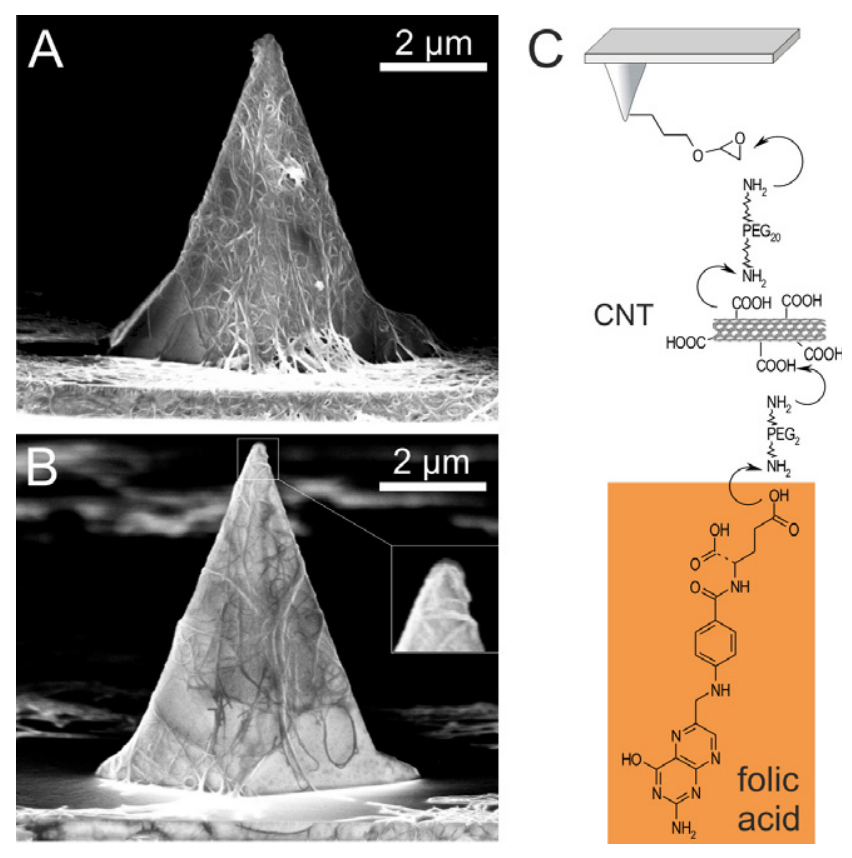

Figure 1. AFM-tip functionalization. ((A), (B)) SEM images of AFM-tips after covalent binding of oxDWNTs and functionalization with folic acid with high and reduced loading of oxDWNTs, respectively. (C) Schematic representation of AFM-tip functionalization in a 5 step process. After epoxy (GPS) coating of the tip PEG-diamine linkers were coupled. Then, oxDWNTs were bound to the linkers via their amine terminus. In step 4 a short PEG-diamine was reacted onto remaining free carboxylic groups on oxDWNT surfaces. Finally, folic acid was bound.

remains zero until the tip reaches the contact point at $\sim 0 \mathrm{~nm}$. With further approach the tip exerts a linearly increasing force on the cell. At contact force of $\sim 200-250 \mathrm{pN}$ the tip movement is reversed. During tip retraction (red, curve) the cantilever relaxes until the force becomes zero again. In case FA has formed a complex with cell surface bound FR, an increasing attractive (negative) force is detected. The force acting on the bond increases up to a critical force value where the receptor-ligand complex dissociates resulting in a jump back to zero force. If no complex is formed, the retraction curve follows closely the approach curve (figure 2(D)).

For the majority of FDCs single and double rupture events were observed (figures 2(A) and (B)), but also multiple consecutive rupture events occurred quite frequently (figure 2(C)). Such events are accompanied by a characteristic nonlinear force signal due to elastic extension of the distensible PEG-linker and the cell, which is clearly visible in the retract part of the FDC in figure 2(A). The observed unbinding length of the individual rupture events were significantly longer then the PEG stretching $(6 \mathrm{~nm})$ itself and varied from a few nanometer up to some micrometer. They were caused by the elastic behavior of the cell and the length of the CNT bundle at the tip apex, whereby the unbinding length was strongly position dependent. The characteristic shape of the cross-linker stretching allows to distinguish specific rupture events of FA-FR complexes from unspecific adhesions [34]. As illustrated in the inset of figure 2(B), the first downward bending (blue) lacks the linker stretching feature (green) and is therefore classified as unspecific adhesion. As an additional specificity proof, T24 cells were exposed to a high concentration $(2 \mu \mathrm{M})$ of FA over night to saturate FA binding receptors [35]. As a result rupture events (figure 2D) were absent in most FDCs. When probing once more FA deprived cells with the same AFM-tip, multiple rupture events were observed again. We note that FDCs with very complex interaction patterns (supporting information figure S1 stacks.i op.org/Nano/25/125704/mmedia) were observed, as well. As those were absent in the specificity proof, they were classified as unspecific adhesion events for sake of simplicity.

\subsection{Statistical analysis of binding/rupture events}

In our study 70 individual cells were probed using in sum 10 different CNT-FA functionalized AFM-tips. For each cell a full dataset of 500-800 FDCs was recorded. The retract parts were analyzed to determine the probability for specific rupture events, and the number of rupture events per FDC (figure 3). Unspecific adhesions were not rated as rupture events. Figure 3(A) shows results for a representative dataset with a high total binding probability of $59 \%$, i.e. $59 \%$ of the FDCs showed one or more specific rupture events in the retract part. In this particular dataset a maximum of seven consecutive events was observed (black columns). For FA saturated cells, probed with the exact same cantilever, the overall binding probability decreased significantly to $7 \%$. Multiple rupture events occurred only occasionally and the maximum number of consecutive events was much lower (red columns). Figures 3(B) and (C) show the averaged binding probability for a total of 49 FA deprived cells (black columns) and 21 FA saturated cells (red columns). In sum 10 functionalized cantilevers were used to collect the data. For FA deprived cells the average binding probability was (37.7 \pm 14.4$) \%$ (mean value \pm standard deviation) with a high frequency of multiple rupture events (figure 3(C)). For cells that were exposed to a high concentration of FA the average binding probability decreased significantly down to $(9.2 \pm 5.2) \%$, and the occurrence of multiple rupture events was notably lower. When probing once more FA deprived cells with the same cantilever, the binding probability returned to the original high level and the frequency of occurrence for multiple ruptures was again increased (see supporting information stacks.iop.or g/Nano/25/125704/mmedia).

When dealing with multiple rupture events in an FDC, careful considerations are required. In case the cantilever jumps back to the baseline between each of the consecutive rupture events (figures 2(B) and (C)) all events can be regarded as independent, and can be analyzed discretely. If the return to the baseline is lacking, it is likely that load is shared between all bonds [36]. This complexity can be avoided by looking only at the very last rupture event in the FDC. The analysis of all last rupture events in a data set of several hundred curves yields a force histogram that can be displayed as a calculated probability density functions (pdf) (figure 4(A)) [18, 37]. The peak maximum in the pdf reflects the most probable rupture force for the ligand-receptor pair.

The most probable rupture force is not a unitary value, instead it depends on the timescale of the measurements [38], 

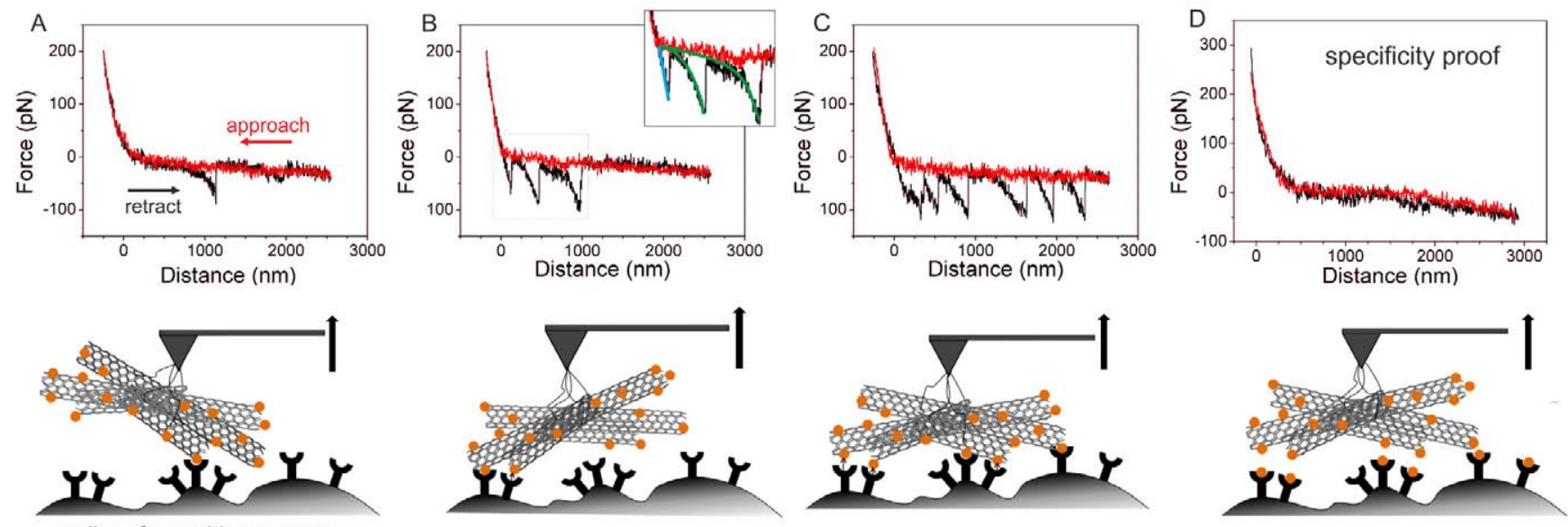

Figure 2. Typical force traces (FDCs) acquired with folate functionalized CNTs on the AFM-tip and folate deprived T24 cells on the surface. Cantilever approach curve is shown in red, retraction in black. Single (A), double (B) and even multiple sequential unbinding events (C) are detected upon retraction of the AFM-tip from the cell (black curve). The bindings disappeared when offering free folate to the cells (D). The cartoons under the force traces illustrate the interaction of folic acid functionalized CNT and the cell surface.
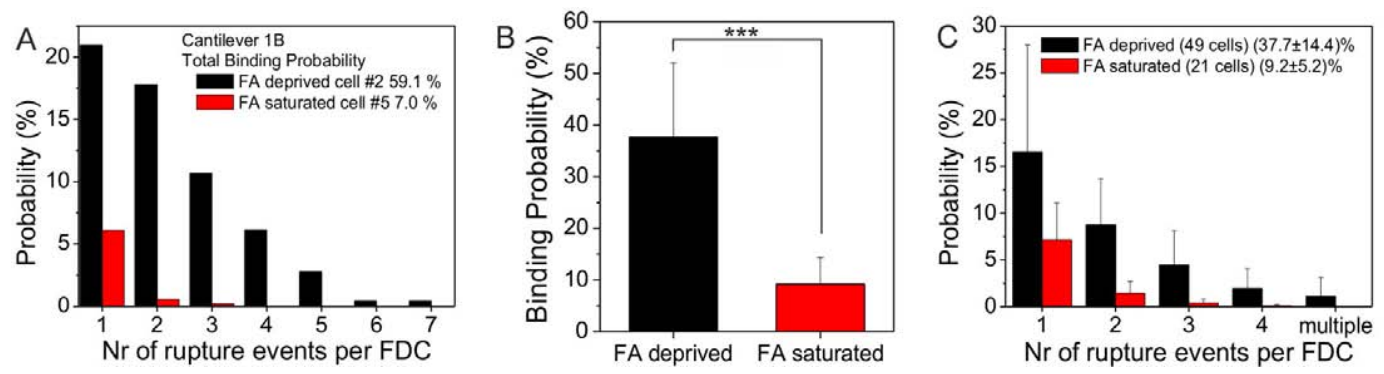

Figure 3. Quantitative comparison of binding probabilities for folate deprived (black columns) and folate saturated (red columns) cancer cells. (A) Representative statistics of one FA deprived cell and one FA saturated cell probed with the same AFM-tip (cantilever 1B), where the total binding probability was $59.1 \%$ for the deprived and $7.0 \%$ for the saturated cell. (B), (C) Summarized statistics for 10 AFM-tips with the total number of FA deprived cells 49 and 21 FA saturated cells. (B) Overall binding probability (mean \pm sd) for FA deprived cells compared to FA saturated cells ( $\left.{ }^{* * *}, p<0.0005\right)$. (C) Probabilities (mean \pm sd) for occurrence of sequential rupture events. Total number $n$ of FDCs evaluated for (B) and (C): $n_{\text {FA deprived cells }}=16.500$ and $n_{\text {FA saturated cells }}=13.000$.

i.e. on the loading rate. The loading rate is the product of effective spring constant $k_{\text {eff }}$ and pulling speed. In the presented experiments, the pulling speed was kept constant at $4000 \mathrm{~nm} \mathrm{~s}^{-1}$ for the $49 \mathrm{FA}$ deprived as well as the 21 FA saturated cells. The effective spring constant $k_{\text {eff }}$, on the other hand, varied for each cell, as $k_{\text {eff }}$ accounts for the combined spring constants of cantilever, linker, and cell properties, e.g. the membrane elasticity at the contact point. The effective spring constant can be determined from the slope of the retract curve at the point of bond rupture [39]. In our experiments we observed low effective spring constants in the range of $0.1 \mathrm{pN} \mathrm{nm}^{-1}$ caused by the softness of the investigated cells. Evaluation of all (last) rupture events results in a pdf for $k_{\text {eff }}$ (figure 4(B)), from which the peak maximum is used to calculate the actual loading rate. As a result the calculated loading rates ranged from 300 to $3000 \mathrm{pN} \mathrm{s}^{-1}$ between all 70 cells (FA deprived and FA saturated). In the basic mathematical model of receptor-ligand interaction in the thermally activated regime $[38,40]$ the measured pull-off force increases logarithmically with the loading rate. According to this theory, the logarithmic fit of the loading rate dependence yields the natural kinetic off-rate $k_{\text {off }}$ that defines the lifetime of the bond in absence of applied force, and the width of the interaction potential $x_{\beta}$. Figure 4(C) shows a plot of the most probable rupture forces against the logarithm of the actual loading rate, where each point represents one of the 70 cells. We find a continuous curve with a linear slope (red curve) that seemingly fits well the theory. However, to allow for a qualified and credible determination of $k_{\text {off }}$ and $x_{\beta}$ the loading rate should be varied over a sufficiently large range that commonly spans at least two orders of magnitude. As the range of loading rates in this experiment is rather limited, we refrain from giving an estimate of kinetic off-rate and width of interaction potential.

\subsection{Internalization of FA functionalized CNTs by T24 cells}

For FA mediated internalization of CNTs we applied a noncovalent functionalization approach using amine terminated 

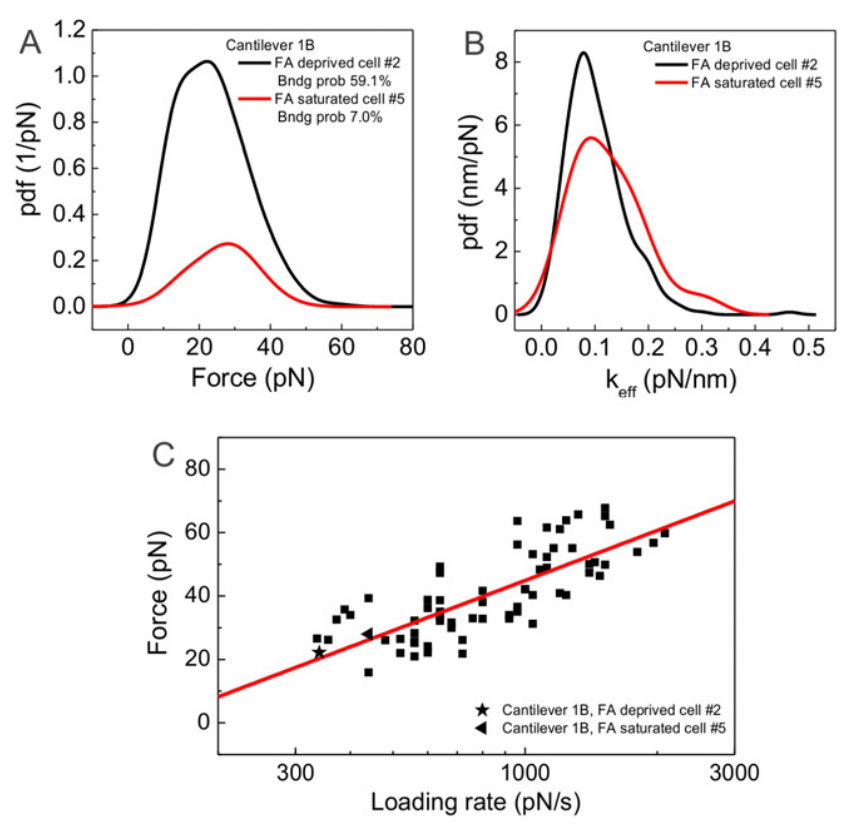

Figure 4. Force spectroscopy. (A) Representative empirical probability density functions (pdfs) of the rupture forces (A) and the effective spring constant $k_{\text {eff }}$ (B) for the cells corresponding to data presented in figure 3(A), black: FA deprived cell, and red: FA saturated cell. (C) Loading rate dependence of the interaction forces in $\log _{10}$ representation and logarithmic fit (red) in accordance with the mathematical model for receptor-ligand interaction in the thermally activated regime by Bell [40] and Evans [38]. Error bars (FWHM of peak maxima in the pdf of $\mathrm{F}$ and $k_{\text {eff }}$, respectively) have been left out in this plot for clarity.

poly(ethyleneglycol) 2000 conjugated phospholipids (DSPE$\mathrm{PEG}_{2000}-\mathrm{NH}_{2}$ ), as it has shown promising for different cancer cell lines [6, 8, 41, 42]. The phospholipid (DSPE, phosphatidylethanolamine) adsorbs onto the CNT surface, while $\mathrm{PEG}_{2000}$ imparts water solubility and prevents non-specific cell uptake. The amino group is used for attachment of FA and fluorescent dye (Alexa647), providing for uncomplicated bi-functionalization (figure $5(\mathrm{G})$ ). In this way functionalized oxDWNT-DSPE-PEG $2000-\mathrm{NH}_{2} /$ Alexa647/FA were incubated to FA deprived and FA saturated T2 4 cells over night. Figure 5 shows representative fluorescence images taken in TIRF configuration. Note that contrast settings for control (figure 5(A)), FA deprived cells (figure 5(B)), and FA saturated cells (figure 5(C)) are identical (fluorescence signal range: 1-2000 counts) to allow for qualitative, as well as semi quantitative comparison of CNT uptake. The images show that FA deprived cells internalized high amounts of CNTs, while uptake by FA saturated cells was considerably lower, thus indicating FA specific CNT uptake by T24 cells.

\subsection{Time traces of internalized CNTs}

Background fluorescence and photobleaching are critical issues for tracking of fluorescently labeled particles. Conventional epi-illumination yields poor contrast, as also out of focus fluorescence is excited. Laser illumination depth in TIRF is below $200 \mathrm{~nm}$, which essentially improves the signal-tobackground ratio, and reduces photobleaching. However, due to restriction of illumination to the cover-slip surface, TIRF is unsuitable for 3D tracking in extended samples. These limitations can be overcome by highly inclined laminated optical sheet (HILO) microscopy [24]. In HILO the laser is adjusted at a sub-critical angle to TIRF. The sample is illuminated with a thin sheet of laser beam, which results in deep illumination, but yields about eight times less background scatter than epi-illumination, thus allowing for single-molecule resolution and 3D tracking in live cells.

In HILO configuration we recorded image sequences of FA deprived T24 after incubation with oxDWNT-DSPE$\mathrm{PEG}_{2000}-\mathrm{NH}_{2} /$ Alexa647/FA over night. Illumination time was set to $5 \mathrm{~ms}$ per frame to avoid rapid photobleaching. Typical sequence lengths ranged between 100 and 200 frames and from 20 to $60 \mathrm{~s}$ depending on the delay between consecutive frames. All cells showed noticeable perinuclear fluorescence from CNT accumulation after over night incubation (figure 6(A)), but also dynamic fluorescence signals were observed moving through the cytosol (supporting information M1, M2 stac ks.iop.org/Nano/25/125704/mmedia). By manually marking fluorescence intensity points in consecutive frames we extracted trajectories and created dynamic diffusion 'roadmaps' (figure 6(B)). We note that only signals in the focal plane contributed to particle trajectories. Although signals leaving the focal plane could still be followed by eye, their fluorescence profile was inevitably diffuse with larger radii and lower intensities. In figure 6(B) we can clearly recognize 'roads' of directed diffusion, but also areas of strict confinement. Also phases of immobilization between segments of directed diffusion ('stop-and-go' diffusion), where CNTs either shortly paused before continuing in the same direction, changed onto another path, or came to a complete halt (supporting information M1, M2 stacks.iop.org/Nano/25/125704/mmedia) were observed.

For analysis of trajectories in the map of figure 6(B) we separated directed from confined diffusion, and looked at the mean square displacement (MSD) in each group. In directed diffusion the MSD exhibits a quadratic time dependence (MSD $\sim t^{2}$ ), while confined diffusion is characterized by a plateau regime. Figure 6(C) shows the ensemble MSD- $t$ plot of 22 directed trajectories (mean $\pm \mathrm{sd}$ ). From the quadratic fit (equation (1), Methods section) a mean speed of $v=$ $0.6 \mu \mathrm{m} \mathrm{s}^{-1}$ was calculated. Figure 6(D) shows the MSD (mean \pm sd) of 43 confined trajectories. The initial slope represents microscopic diffusion within the confinement zone. From the analytical expression in equation (2) (Methods section) we determined a corral size $R$ of $\sim 211 \mathrm{~nm}$ for the confinement structure and a diffusion constant $D$ of $0.0035 \mu \mathrm{m}^{2} \mathrm{~s}^{-1}$ within the corral.

Finally, due to the frequent observation of stop and go diffusion in conjunction with directed motion we started to suspect active transport of FA functionalized CNTs along cell filaments such as microtubules and possible involvement of microtubule motors [43]. In order to test this hypothesis we treated oxDWNT-DSPE-PEG $2000-\mathrm{NH}_{2} / \mathrm{Alexa647/FA}$ incubated T24 cells with nocodazole, a microtubule depolymerizing agent. We found that the directed diffusion was completely abolished (figures 6(E), (F), supporting information M3, M4 stacks.iop.org/Nano/25/125704/mmedia). 

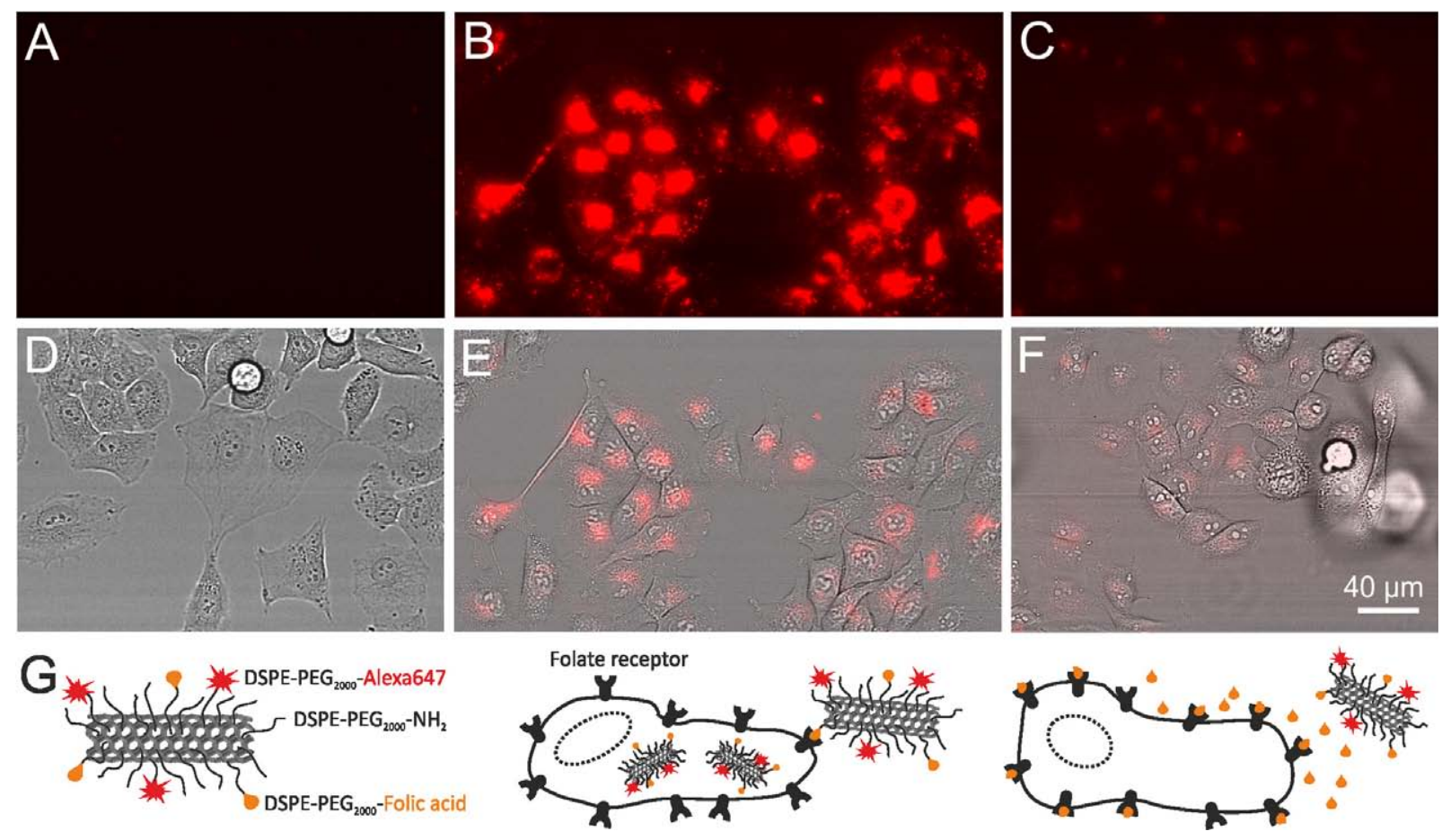

Figure 5. Specific uptake of FA functionalized oxDWNTs by T24 cells. (A)-(C) TIRF microscopy images (contrast: counts [0, 2000]). ((D)-(F)) Corresponding overlay of bright-field and TIRF images (unequal contrast). ((A), (D)) Control cells without exposure to CNTs. ((B), (E)) FA deprived cells after $12 \mathrm{~h}$ incubation with CNTs. ((C), (F)) FA deprived cells were supplemented with a high dose of FA starting $6 \mathrm{~h}$ prior to the $12 \mathrm{~h}$ incubation with CNTs. All images at same scale with scale bar $40 \mu \mathrm{m}$. (G) Schematic illustration of fluorescently labeled FA functionalized CNTs for targeting of FR expressing cells. Blocking of FR by free FA inhibits specific uptake of FA functionalized CNTs.

\section{Discussion}

The aim of this study was to elucidate new aspects of FA mediated uptake of CNTs by looking at their selective binding, internalization, and intracellular transport on a single nanotube level. For our experiments we chose DWNTs, because their properties make them particularly attractive as delivery platform. Chemical oxidation, as a popular method that renders CNTs water soluble and provides reactive sites for covalent chemistry, disrupts the nanotube surface [25]. For oxDWNTs we expect that primarily the outer nanotube is effected, while mechanical and electronic properties of the inner tube remain unaltered [44]. Also, reactive carboxyl groups may be present along the entire nanotube in contrast to oxidized single walled carbon nanotubes (SWNTs), which tend to break at surface defects leading to short tubes with $\mathrm{COOH}$ groups mainly at their ends.

In MRFS we exploited the carboxyl groups on the surface of oxDWNTs to stably attach nanotubes to tips of AFM cantilevers and functionalize tip-bound CNTs with FA. We were able to show binding of FA functionalized CNTs to FA binding receptors on live cancer cells. The specificity of this interaction was proven though exposure of cells to high concentrations of free FA. FA binding receptors were blocked and binding events with CNT-FA functionalized AFM-tips were absent. We further determined rupture forces between CNT bound FA and FR, and found a logarithmic dependence of the rupture force with increasing loading rate.
This behavior is in accordance with a well established theory for receptor-ligand interaction, proving once more specificity of the observed interactions. Although our herein presented approach was limited to the point that we were not able to ensure the attachment of only a single nanotube to the AFM-tip apex, we could prove specific interaction of CNT bound FA with FR on the surface of cancer cells. This demonstrates the benefit of AFM based MRFS as a potential screening tool in the design of targeted nanoparticle systems in the growing field of nanomedicine [45].

However, AFM is foremost a surface technique and offers almost no information about CNTs internalization by the cell [46]. Several groups have investigated uptake and intracellular distribution of FA functionalized and fluorescently labeled CNTs using laser scanning confocal microscopy (LSCM) [4, 6-9]. Likewise we have employed fluorescence microscopy in TIRF configuration to confirm uptake of non-covalently functionalized oxDWNT FA conjugates. Evidently internalization of oxDWNT-DSPE-PEG 2000 $\mathrm{NH}_{2} /$ Alexa647/FA entities could be inhibited by competitive binding of FA in cells, which were exposed to high concentrations of free FA prior and during incubation with nanotubes. This confirmed receptor specific, i.e. FA mediated uptake of oxDWNT-DSPE-PEG $2000-\mathrm{NH}_{2}$ /Alexa647/FA conjugates on one hand, and sufficient inhibition of non-specific uptake of nanotubes on the other. The inhibition experiments further showed that oxDWNT-DSPE-PEG $2000-\mathrm{NH}_{2} /$ Alexa647/FA conjugates were taken up rather as entire complexes, and 

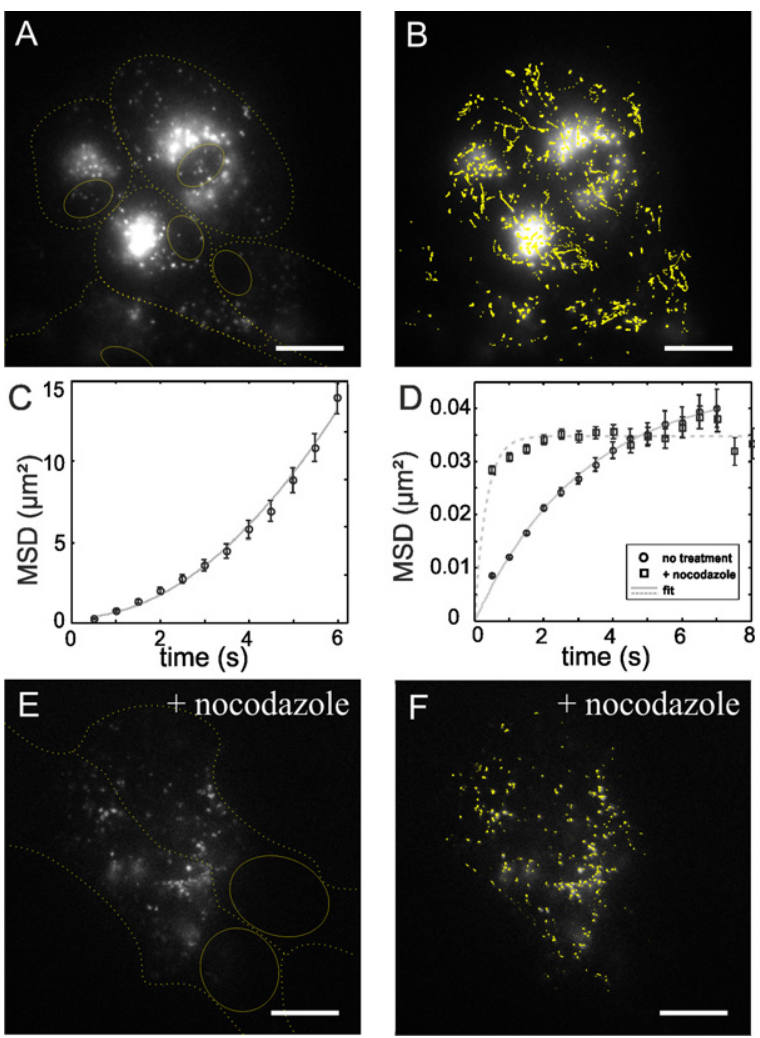

Figure 6. Single-particle tracking in FA deprived cells after $12 \mathrm{~h}$ incubation with FA functionalized CNTs. All scale bars $17 \mu \mathrm{m}$. (A) HILO fluorescence image of a representative cell group. Dashed lines mark cell borders; solid lines mark nuclei (see supporting information M1 stacks.iop.org/Nano/25/125704/mmedia).

(B) Overlay of HILO image (A) with corresponding point-map of $x$-, $y$-positions of mobile CNTs (yellow). (C) MSD- $t$ plot for 22 directed trajectories extracted from (B). The quadratic fit yields a mean speed of $v=0.6 \mu \mathrm{m} \mathrm{s}^{-1}$. (D) MSD- $t$ plots for 43 confined trajectories extracted from (B) (circles) and 41 traces from nocodazole treated cells (F) (squares). The fit for untreated cells yields a corral size of $\sim 211 \mathrm{~nm}$ with diffusion constant of $0.0035 \mu \mathrm{m}^{2} \mathrm{~s}^{-1}$ within the confinement zone. Treatment with nocodazole leads to loss of CNT mobility $((\mathrm{E}),(\mathrm{F}))$ (see supporting information M3 stacks.iop.org/Nano/25/125704/mmedia), which is reflected in the reduced corral size of $\sim 187 \mathrm{~nm}$ and lower micro domain diffusion constant of $0.0026 \mu \mathrm{m}^{2} \mathrm{~s}^{-1}$. For detailed discussion see text.

that fluorescence signals inside the cells were not a result of free DSPE-PEG 2000 -Alexa647 or fragmented PEG-Alexa647 molecules [8].

We observed that FR specific uptake of oxDWNT- DSPE$\mathrm{PEG}_{2000}-\mathrm{NH}_{2} /$ Alexa647/FA entities in T24 cells depended strongly on the incubation time. Twelve hours incubation finally appeared well suited to determine the fate of internalized CNTs and follow cellular dynamics at the same time (supporting information figure S6 stacks.iop.org/Nano/25/12 $5704 /$ mmedia). We found that most CNTs had accumulated around the nucleus after $12 \mathrm{~h}$ of exposure in accordance with previous reports for cellular uptake of FA functionalized CNTs $[6,7]$. As there is currently no consensus about translocation of CNTs across the nuclear membrane [47], it is worth mentioning that our investigations showed no fluorescence from inside the nuclei (supporting information figure S6 stacks .iop.org/Nano/25/125704/mmedia), suggesting lack of nuclear translocation of oxDWNT-DSPE-PEG $2000-\mathrm{NH}_{2} / \mathrm{Alexa647/FA}$ complexes [42].

To enable time-dependent three-dimensional tracking of individual CNTs in the cytosol fluorescence illumination was changed from TIRF to HILO illumination. Such advanced fluorescence methods are still technically demanding and require specialized setups, as well as precise control of illumination protocols. Therefore, only very little quantitative data on CNT dynamics inside cells is available, such as the work of Jin et al [48, 49], who have investigated uptake and intracellular transport of SWNTs in a non-targeted approach.

In our sequences we observed mainly directed traffic of CNTs towards and away from the nuclear region in association with a characteristic 'stop-and-go' diffusion. We also found trajectories of strict confinement in the cytosol. Directed diffusion with partial exhibition of 'stop-and-go' phases, as well as areas of local confinement has also been reported by Jin et al [48,49]. Our analysis of the confinement yielded a corral size of $211 \mathrm{~nm}$ with a diffusion constant of $0.0035 \mu \mathrm{m}^{2} \mathrm{~s}^{-1}$, while analysis of the directed motion of oxDWNT-DSPE-PEG $2000-\mathrm{NH}_{2} /$ Alexa647/FA in the cytosol showed a mean speed of $0.6 \mu \mathrm{m} \mathrm{s}^{-1}$. These values compare with the lower ranges of corral sizes and velocities reported by Jin and co-workers [49], as the researchers observed great variability of confinement structures up to the $\mu \mathrm{m}$ regime and found velocities between 0.3 and $2.3 \mu \mathrm{m} \mathrm{s}^{-1}$ for directed diffusion of SWNT in the cytosol. It is possible that comparably low diffusion velocities and small corral sizes reflect an increased size and naturally reduced mobility of oxDWNT-DSPE-PEG $2000-\mathrm{NH}_{2}$ /Alexa647/FA complexes compared to smaller SWNTs in Jin's study (supporting information figure S7 stacks.iop.org/Nano/25/125704/mmedia).

Consistencies with in vitro assays of individual kinesin motors, which moved along microtubule filaments with velocities of 0.6 to $0.8 \mu \mathrm{m} \mathrm{s}^{-1}$ [50], and a study of QD-tagged kinesin [51], which yielded an average velocity of $0.57 \pm$ $0.02 \mu \mathrm{m} \mathrm{s}^{-1}$, lead Jin and co-workers to speculate that motor proteins might be involved in an active transport of nanotubes in the cytosol. Our observations that treatment of the cell with nocodazole suppresses the intracellular transport of oxDWNTDSPE-PEG $2000-\mathrm{NH}_{2} /$ Alexa647/FA, as well as the frequent stop and go diffusion, fit well with this picture. Nevertheless, to unravel the intracellular transport mechanism of CNTs additional controls and specific perturbation experiments are indispensible and will be included in follow up studies.

\section{Conclusion}

We believe single-molecule techniques such as MRFS and SPT and their combination has potential for comparative investigations of different drug-delivery systems and targeting agents. Especially dynamic aspects of particle uptake and intracellular transport could play an important role in the evaluation of efficacy of targeting approaches and the intracellular delivery of payloads through nano-carriers for future therapies. 


\section{Acknowledgments}

This work was supported by EC grant Marie Curie RTN-CT2006-035616, CARBIO 'Carbon nanotubes for biomedical applications' and Austrian FFG grant mnt-era.net 823980, 'IntelliTip'. We thank Dr Josef Madl (BIOSS Freiburg, Germany) and Dr Julian Weghuber (FH Wels, Austria) for their expertise and helpful discussions.

\section{References}

[1] Kostarelos K et al 2007 Nature Nanotechnol. 2 108-13

[2] Liu Z, Tabakman S, Welsher K and Dai H J 2009 Nano Res. 2 85-120

[3] Heister E et al 2009 Carbon 47 2152-60

[4] Shi X Y et al 2009 Biomacromolecules 10 1744-50

[5] Hutter J L and Bechhoefer J 1993 Rev. Sci. Instrum. 64 1868-73

[6] Kam N W and Dai H 2005 J. Am. Chem. Soc. 127 6021-6

[7] Kang B, Yu D C, Chang S Q, Dai Y D and Ding Y 2008 Nanotechnology 19375103

[8] Zeineldin R, Al-Haik M and Hudson L G 2009 Nano Lett. 9751-7

[9] Dhar S, Liu Z, Thomale J, Dai H J and Lippard S J 2008 J. Am. Chem. Soc. 130 11467-76

[10] Zhang X K, Meng L J, Lu Q G, Fei Z F and Dyson P J 2009 Biomaterials 30 6041-7

[11] Holt B D, Dahl K N and Islam M F 2012 ACS Nano 6 3481-90

[12] Leamon C P and Low P S 1991 Proc. Natl Acad. Sci. USA 88 5572-6

[13] Lu Y J, Sega E, Leamon C P and Low P S 2004 Adv. Drug Deliv. Rev. 56 1161-76

[14] Garcia-Bennett A, Nees M and Fadeel B 2011 Biochem. Pharmacol. 81 976-84

[15] Russell-Jones G, McTavish K, McEwan J, Rice J and Nowotnik D 2004 J. Inorg. Biochem. 98 1625-33

[16] Hinterdorfer P and Dufrene Y F 2006 Nat. Meth. 3 347-55

[17] Pfister G et al 2005 J. Cell Sci. 118 1587-94

[18] Rankl C et al 2008 Proc. Natl. Acad. Sci. USA 106 17778-83

[19] Zhu R et al 2013 J. Am. Chem. Soc. 135 3640-6

[20] Saxton M J and Jacobson K 1997 Annu. Rev. Biophys. Biomol. Struct. 26 373-99

[21] Levi V and Gratton E 2007 Cell Biochem. Biophys. 48 1-15
[22] Schutz G J, Sonnleitner M, Hinterdorfer P and Schindler H 2000 Mol. Membr. Biol. 17 17-29

[23] Wieser S and Schütz G J 2008 Methods 46 131-40

[24] Tokunaga M, Imamoto N and Sakata-Sogawa K 2008 Nature Methods 5 159-61

[25] Heister E, Neves V, Lamprecht C, Silva S R P, Coley H M and McFadden J 2012 Carbon 50 622-32

[26] Butt H J and Jaschke M 1995 Nanotechnology 6 1-7

[27] Lamprecht C, Torin Huzil J, Ivanova M V and Foldvari M 2011 Drug Deliv. Lett. 1 45-57

[28] Saxton M J 1994 Biophys. J. 67 2110-9

[29] Ebner A et al 2008 Top. Curr. Chem. 285 29-76

[30] Wildling L et al 2011 Bioconjug. Chem. 22 1239-48

[31] Osswald S, Flahaut E and Gogotsi Y 2006 Chem. Mater. 18 1525-33

[32] Jhaveri M S, Wagner C and Trepel J B 2001 Mol. Pharmacol. 60 1288-95

[33] Kane M A et al 1988 J. Clin. Investig. 81 1398-406

[34] Hinterdorfer P, Baumgartner W, Gruber H J, Schilcher K and Schindler H 1996 Proc. Natl Acad. Sci. USA 93 3477-81

[35] Kamen B A and Capdevila A 1986 Proc. Natl Acad. Sci. USA 83 5983-7

[36] Williams P M 2003 Anal. Chim. Acta 479 107-15

[37] Baumgartner W, Hinterdorfer P and Schindler H 2000 Ultramicroscopy 82 85-95

[38] Evans E and Ritchie K 1997 Biophys. J. 72 1541-55

[39] Evans E and Ritchie K 1999 Biophys. J. 76 2439-47

[40] Bell G I 1978 Science 200 618-27

[41] Liu Z et al 2007 Nature Nanotechnol. 2 47-52

[42] Lamprecht C et al 2012 J. Phys.: Condens. Matter 24164206

[43] Nan X L, Sims P A, Chen P and Xie X S 2005 J. Phys. Chem. B 109 24220-4

[44] Flahaut E, Bacsa R, Peigney A and Laurent C 2003 Chem. Commun. 12 1442-3

[45] Oliveira H, Rangl M, Ebner A, Mayer B, Hinterdorfer P and Pêgo A P 2011 Small 7 1236-41

[46] Lamprecht C et al 2009 Nanotechnology 20434001

[47] Mu Q X, Broughton D L and Yan B 2009 Nano Lett. 9 4370-5

[48] Jin H, Heller D A, Sharma R and Strano M S 2009 ACS Nano 3 149-58

[49] Jin H, Heller D A and Strano M S 2008 Nano Lett. 8 1577-85

[50] Friedman D S and Vale R D 1999 Nature Cell Biol. 1 293-7

[51] Courty S, Luccardini C, Bellaiche Y, Cappello G and Dahan M 2006 Nano Lett. 6 1491-5 